\title{
Correlation between small intestinal bacterial overgrowth and irritable bowel syndrome and the prognosis of treatment
}

\author{
Xuelian Yu ${ }^{1,2 \#}$, Yue Li ${ }^{1,2 \#}$, Feng Xiang ${ }^{2,3}$, Jingjuan Feng ${ }^{2,3}$ \\ ${ }^{1}$ Department of Geriatric Medicine, Sichuan Provincial People's Hospital, University of Electronic Science and Technology of China, Chengdu, \\ China; ${ }^{2}$ Chinese Academy of Sciences Sichuan Translational Medicine Research Hospital, Chengdu, China; ${ }^{3}$ Department of Anorectal Surgery, \\ Sichuan Provincial People's Hospital, University of Electronic Science and Technology of China, Chengdu, China \\ Contributions: (I) Conception and design: X Yu, Y Li; (II) Administrative support: F Xiang, J Feng; (III) Provision of study materials or patients: All \\ authors; (IV) Collection and assembly of data: All authors; (V) Data analysis and interpretation: All authors; (VI) Manuscript writing: All authors; (VII) \\ Final approval of manuscript: All authors. \\ "These authors contributed equally to this work. \\ Correspondence to: Feng Xiang; Jingjuan Feng. Department of Anorectal Surgery, Sichuan Provincial People's Hospital, University of Electronic \\ Science and Technology of China, No. 32, Section 2, West First Ring Road, Chengdu 610072, China. Email: xiangfeng20210105@163.com; \\ fengjingjuan2021@163.com.
}

Background: The aim of the present study was to analyze the relationship between small intestinal bacterial overgrowth (SIBO) and irritable bowel syndrome (IBS) and the prognosis of treatment.

Methods: A total of127 IBS patients (IBS group) were selected, and based on the results of the methane/ hydrogen breath test, they were divided into SIBO-positive group (subdivided into the antibiotic group, microecological group, and placebo group according to difference in treatment methods) and the SIBOnegative group. The incidence of SIBO and inflammatory factors in different populations were compared. Moreover, differences in the negative conversion rate of intestinal bacterial overgrowth, symptom improvement, interleukin (IL)-1 $\beta$, and IL-10 levels in the antibiotic group, microecological group, and placebo group before and after treatment were compared.

Results: The IL-1 $\beta$ level of the IBS group was higher than that of the control group, but the level of IL-10 was lower than that of the control group $(\mathrm{P}<0.05)$. The level of IL-1 $\beta$ in the SIBO-positive group was higher than that in the SIBO-negative group, while IL-10 level was lower in the SIBO-positive group compared with the SIBO-negative group $(\mathrm{P}<0.05)$. Symptom scores after treatment in the antibiotic group and the microecological group were lower than those in the same groups before treatment and those in the placebo group after treatment $(\mathrm{P}<0.05)$. After treatment, the level of IL-1 $\beta$ in the antibiotic group and the microecological group decreased, whereas the level of IL-10 increased $(\mathrm{P}<0.05)$. Furthermore, the total clinical effective rate and negative conversion rate of the antibiotic group and the microecological group were higher than those of the placebo group $(\mathrm{P}<0.05)$; however, the comparison of symptom scores, inflammatory factors, and total clinical effective rate before and after treatment in the placebo group was not statistically significant $(\mathrm{P}<0.05)$. The symptom score after treatment in the negative conversion group was lower than that in the non-negative conversion group $(\mathrm{P}<0.05)$.

Conclusions: SIBO plays an important role in the occurrence of IBS. Antibiotics and microecological agents can be used to correct SIBO in clinical practice to improve the symptoms of IBS patients and peripheral blood inflammatory factors.

Keywords: Small intestinal bacterial overgrown; irritable bowel syndrome (IBS); prognosis; methane/hydrogen breath test

Submitted Jan 28, 2021. Accepted for publication Mar 12, 2021.

doi: $10.21037 /$ apm-21-427

View this article at: http://dx.doi.org/10.21037/apm-21-427 


\section{Introduction}

Irritable bowel syndrome (IBS), the most common gastrointestinal dysfunction in clinical practice, is characterized by abdominal pain, bloating, and changes in bowel habits. It is a clinical and social issue that warrants attention (1). The pathogenesis and mechanism of IBS have not yet been fully understood. Current research on the pathophysiological basis of IBS suggests that its pathogenesis is related to abnormal gastrointestinal motility, mental factors, immune abnormalities, and infections (2). Small intestinal bacterial overgrown (SIBO) refers to a syndrome of malabsorption of various nutrients caused by small intestinal stasis and excessive bacterial growth due to organic or functional reasons. Its clinical manifestations include abdominal pain, bloating, constipation, diarrhea, and/or malabsorption (3). In recent years, studies have found that the rate of positive SIBO in IBS patients is significantly increased, and it has been found that eradicating overgrown small intestinal bacteria can improve the symptoms of IBS patients. Therefore, the relationship between SIBO and IBS has attracted widespread attention $(4,5)$. In the present study, we analyzed the correlation between SIBO and IBS onset and treatment prognosis, aiming to provide ideas for clinical treatment. We present the following article in accordance with the STROBE reporting checklist (available at http://dx.doi.org/10.21037/apm-21-427).

\section{Methods}

\section{General information}

A total of 127 patients with IBS admitted to Sichuan Provincial People's Hospital, university of electronic science and technology of china from July 2018 to July 2019 were selected as the IBS group. Of these, 69 were males and 56 were females, with an age range of from 26-72 years and an average age of $49.71 \pm 12.32$ years. Forty-five cases had diarrhea-type IBS, 42 cases had constipation-type IBS, and 38 cases had alternating diarrhea and constipation. All patients agreed to participate in this study and signed an informed consent form. This study was approved by the Sichuan Provincial People's Hospital, University of Electronic Science and Technology of China (No. 20180529). The study was conducted in accordance with the Declaration of Helsinki (as revised in 2013).

Inclusion criteria were as follows: (I) those who meet the Rome III diagnostic criteria (6): abdominal pain or discomfort recurring in the past 6 months, frequency of at least 2 days a week, and symptoms appear on at least 3 days a month in the past 3 months. As well as this, 2 or more of the symptoms occurred: symptoms improved after defecation; changes in the frequency of bowel movements during the attack (defecation $\geq 3$ times a day or $\leq 3$ times a week); stool trait (appearance) changes at onset; (II) no organic diseases of the digestive system were found after the physical examination, blood biochemistry, digestive endoscopy, or related imaging examinations; and (III) clinical data were complete. Exclusion criteria were as follows: (I) accompanied by other organic diseases that can cause abnormal gastrointestinal function, such as diabetes, thyroid disease, and connective tissue disease; (II) severe heart, lung, liver, or kidney disease; (III) previous history of abdominal surgery; (IV) use of antibiotics, lactulose, antacids, or drugs that affect gastrointestinal motility 4 weeks before study enrollment; (V) barium meal radiography and enema 1 week before study enrollment; and (VI) history of acute enteritis.

At the same time, 90 healthy controls who agreed to participate in the trial were selected as the control group. Of these, 53 were males and 37 were females, with an age range of $28-70$ years and an average age of $49.63 \pm 12.33$ years. There were no differences in general patient characteristics between 2 groups $(\mathrm{P}>0.05)$.

\section{Observational indicators and methods}

\section{Methane/hydrogen breath test}

The HHBT-01 hydrogen breath tester (Shenzhen CNNC Headway Biotechnology, Shenzhen, China) is employed in this experiment. The hydrogen production food was prohibited for $24 \mathrm{~h}$ before the test, and the patients fasted for $12 \mathrm{~h}$ before the test. The patients were instructed to brush their teeth and rinse their mouth prior to the examination, and remained awake and quiet during the test. The patients avoided vigorous exercise to avoid bowel movements and fluctuations in lung ventilation.

The experiments were performed before and after treatment, respectively. Before the test, the HHBT-01 hydrogen breath tester operating instructions were strictly followed to combine the breath hydrogen tester. The breath tube of the hydrogen micro-analyzer was used to blow into and the flow rate was controlled at about $250 \mathrm{~mL} / \mathrm{min}$. After the exhaled gas was dried, it was captured between the sensor and the receiver valve, and the reading at this time was recorded as the concentration of hydrogen in the patient's exhaled breath. Basic expiratory hydrogen was remeasured every minute, and if it was still high, the test was 
Table 1 Comparison of interleukin (IL)-1 $\beta$ and IL-10 levels between the irritable bowel syndrome (IBS) group and control group $(\bar{x} \pm \mathrm{S}, \mathrm{pg} / \mathrm{mL})$

\begin{tabular}{lcc}
\hline Group & $\mathrm{IL}-1 \beta$ & $\mathrm{IL}-10$ \\
\hline IBS group $(\mathrm{n}=127)$ & $80.45 \pm 4.63$ & $35.78 \pm 5.67$ \\
Control group $(\mathrm{n}=90)$ & $21.63 \pm 3.05$ & $137.59 \pm 15.83$ \\
$t$-test & 105.369 & 66.740 \\
$P$ value & $<0.001$ & $<0.001$ \\
\hline
\end{tabular}

continued. If it dropped significantly, the test was performed on another day, as this could be due to insufficient food before the test or food that was slow to digest. At the beginning of the experiment, the lactulose oral solution package (10 g/package) was dissolved in $180-240 \mathrm{~mL}$ warm water for oral administration and drunk within $2 \mathrm{~min}$. After taking lactulose, the expiratory hydrogen content was measured and recorded every $20 \mathrm{~min}$ for a total of $2 \mathrm{~h}$. Finally, the expiratory hydrogen concentration curve was drawn and analyzed. Positive diagnostic criteria were fasting basal expiratory $\mathrm{H} 2 \geq 20 \mathrm{ppm}$ and repeated determinations every minute (all H2 $\geq 20 \mathrm{ppm}$ ) (7). Or according to the following standards: (I) obvious doublepeak pattern, namely the early small intestine peak and the late colon peak; and (II) the time from the start of hydrogen production after taking lactulose was $<90 \mathrm{~min}$, and the absolute value of the increase in hydrogen concentration was $>20 \mathrm{ppm}$.

\section{Detection of interleukin (IL)-1ß and IL-10}

In total, $3 \mathrm{~mL}$ of fasting venous blood extracted in the morning was centrifuged $(1,088 \mathrm{~g}, 5 \mathrm{~min}$, centrifugal radius $8 \mathrm{~cm}$ ), and serum was then separated. IL- $1 \beta$ and IL-10 were measured by enzyme-linked immunosorbent assay method. The double-antibody sandwich kit (Beijing Yiming Biotechnology, Beijing, China) was used to detected protein expressive levels of IL-1 $\beta$ and IL-10, and the operation was carried out in strict accordance with the kit test instructions.

\section{Symptom score}

The following 6items were scored separately according to severity: occurrence of abdominal pain in IBS (times/week), the time of abdominal pain occurrence (h/day), presence or absence of abdominal distension, and abdominal pain during defecation, abnormal rate of defecation frequency $(d / w)$, rate of abnormal defecation characteristics, and rate of mucus. The sum of the scores of each item was the symptom score (8). The scores were recorded for the symptoms before and after treatment, and the treatment effect was determined according to the changes in the symptom score.

\section{Efficacy determination}

Efficacy standards were as follows: (I) significantly effective: all clinical discomfort symptoms disappear, the stool was 1-2 times/day, soft stools, shaped, and no mucus; (II) effective: some symptoms disappear or more than half of the above symptoms improve; (III) ineffective: symptoms did not significantly improve after treatment. Total effective rate $=$ significantly effective rate + effective rate (9).

\section{Statistical analysis}

SPSS version 22.0 (IBM, Armonk, NY, USA) was used to analyze the data in the present study. Measurement data were expressed as mean \pm standard deviation $(\bar{x} \pm S)$; $t$-test was used for comparisons between 2 groups, and F-test was used for comparisons between multiple groups. Count data were expressed as percentage (\%) using the $\chi^{2}$-test; $\mathrm{P}<0.05$ indicated that the difference was statistically significant.

\section{Results}

\section{Comparison of IL-1ß and IL-10 levels between the IBS group and the control group}

The level of IL-1 $\beta$ in the IBS group was higher than that of the control group, but the level of IL-10 was lower than that of the control group (Table 1).

\section{Comparison of the incidence of SIBO between the IBS group and the control group}

The positive rate of the SIBO test in the IBS group was $79.53 \%$, and the positive rate of the SIBO test in the control group was $16.67 \%$. The positive rate of the SIBO test in the IBS group was higher than that in the control group, and the difference between these 2 groups was statistically significant $(\mathrm{P}<0.05)$, as shown in Table 2 . The 101 positive patients were divided into 3 groups according to the difference in treatment methods as follows: antibiotic group ( $n=39)$, microecological group $(n=36)$, and placebo group $(n=26)$. 
Table 2 Comparison of the incidence of small intestinal bacterial overgrowth (SIBO) between the irritable bowel syndrome (IBS) group and the control group [n (\%)]

\begin{tabular}{lcc}
\hline Group & SIBO positive & SIBO negative \\
\hline IBS group $(\mathrm{n}=127)$ & $101(79.53)$ & $26(20.47)$ \\
Control group $(\mathrm{n}=90)$ & $15(16.67)$ & $75(83.33)$ \\
$\chi^{2}$-test & \multicolumn{2}{c}{83.654} \\
$\mathrm{P}$ value & \multicolumn{2}{c}{$<0.001$} \\
\hline
\end{tabular}

Table 3 Comparison of interleukin (IL)-1 $\beta$ and IL-10 levels between small intestinal bacterial overgrowth (SIBO)-positive group and SIBO-negative group $(\bar{x} \pm \mathrm{S}, \mathrm{pg} / \mathrm{mL})$

\begin{tabular}{lcc}
\hline Group & $\mathrm{IL}-1 \beta$ & $\mathrm{IL}-10$ \\
\hline SIBO-positive group $(\mathrm{n}=101)$ & $83.36 \pm 2.50$ & $30.63 \pm 2.81$ \\
SIBO-negative group $(\mathrm{n}=26)$ & $78.01 \pm 1.63$ & $41.08 \pm 2.73$ \\
$t$-test & 10.344 & 17.006 \\
$\mathrm{P}$ value & $<0.001$ & $<0.001$ \\
\hline
\end{tabular}

Comparison of $I L-1 \beta$ and $I L-10$ levels between the SIBOpositive group and the SIBO-negative group

The IL-1 $\beta$ level of the SIBO-positive group was higher than that of the SIBO-negative group, whereas the IL-10 level was lower than that of the SIBO-negative group. The difference was statistically significant $(\mathrm{P}<0.05)$, as shown in Table 3.

\section{Comparison of symptom scores in the antibiotic group, microecological group, and placebo group before and after the intervention}

The symptom scores of the 3 groups before treatment were not statistically significantly different $(\mathrm{P}>0.05)$. The symptom scores of the antibiotic group and the probiotic group after treatment were significantly lower than those of the same groups before treatment, and the difference was statistically significant $(\mathrm{P}<0.05)$. After treatment, symptom scores in the antibiotic group and the microecological group were lower than those in the placebo group $(\mathrm{P}<0.05)$, but the difference between the 2 groups was not statistically significant $(\mathrm{P}>0.05)$. Moreover, the symptom scores in the placebo group before and after treatment were not statistically significantly different $(\mathrm{P}>0.05)$ (Table 4).
Table 4 Comparison of symptom scores in the antibiotic group, microecological group, and placebo group before and after intervention $(\bar{x} \pm S$, points $)$

\begin{tabular}{lcc}
\hline Group & Before treatment & After treatment \\
\hline Antibiotic group $(\mathrm{n}=39)$ & $11.95 \pm 3.56$ & $4.03 \pm 1.05^{\mathrm{ab}}$ \\
Microecological group $(\mathrm{n}=36)$ & $11.84 \pm 3.51$ & $4.16 \pm 1.01^{\mathrm{ab}}$ \\
Placebo group $(\mathrm{n}=26)$ & $11.86 \pm 3.89$ & $10.99 \pm 3.94$ \\
$\mathrm{~F}$ & 0.01 & 96.69 \\
$\mathrm{P}$ value & 0.990 & $<0.001$ \\
\hline${ }^{\mathrm{a}}$, compared with the same group before treatment $(\mathrm{P}<0.05) ;$ \\
${ }^{\mathrm{b}}$, compared with the placebo group after treatment $(\mathrm{P}<0.05)$.
\end{tabular}

Comparison of IL-1ß and IL-10 levels in the antibiotic group, microecological group, and placebo group before and after the intervention

The levels of IL-1 $\beta$ and IL-10 before treatment in the 3 groups were not statistically significantly different $(\mathrm{P}>0.05)$. After treatment, the level of IL- $1 \beta$ decreased and the level of IL-10 increased in the antibiotic group and microecological group; the difference was statistically significant $(\mathrm{P}<0.05)$; however, the comparison between the 2 groups was not statistically significant $(P>0.05)$; The levels of IL-1 $\beta$ and IL-10 in the placebo group were not statistically significant before and after treatment $(\mathrm{P}>0.05)$ (Table 5).

\section{Comparison of the intervention efficacy in the antibiotic group, microecological group, and placebo group}

The total clinical effective rate of the antibiotic group and the microecological group was higher than that of the placebo group; the difference was statistically significant $(\mathrm{P}<0.05)$, but the total clinical effective rate between the antibiotic group and the microecological group was not statistically significantly different $(\mathrm{P}>0.05)$ (Table 6).

\section{Negative conversion rate of patients in the antibiotic group, microecological group, and placebo group}

The negative conversion rate of the antibiotic group and the microecological group was higher than that of the placebo group; the difference was statistically significant $(\mathrm{P}<0.05)$, while the total clinical effective rate between the antibiotic group and the microecological group was not statistically significantly different $(\mathrm{P}>0.05)$, as shown in Table 7 . 
Table 5 Comparison of interleukin (IL)-1 $\beta$ and IL-10 levels before and after intervention in the antibiotic group, microecological group, and placebo group $(\bar{x} \pm \mathrm{S}, \mathrm{pg} / \mathrm{mL})$

\begin{tabular}{|c|c|c|c|c|}
\hline Group & \multicolumn{2}{|c|}{$\mathrm{IL}-1 \beta$} & \multicolumn{2}{|c|}{ IL-10 } \\
\hline Antibiotic group ( $\mathrm{n}=39$ ) & $81.63 \pm 1.56$ & $26.09 \pm 1.09^{\mathrm{ab}}$ & $31.05 \pm 2.60$ & $43.09 \pm 2.53^{\mathrm{ab}}$ \\
\hline Microecological group $(n=36)$ & $81.60 \pm 1.23$ & $26.29 \pm 1.11^{\mathrm{ab}}$ & $31.22 \pm 2.69$ & $43.15 \pm 2.50^{\mathrm{ab}}$ \\
\hline Placebo group $(n=26)$ & $81.69 \pm 1.25$ & $80.63 \pm 1.20$ & $31.25 \pm 2.61$ & $32.09 \pm 2.59$ \\
\hline$P$ value & 0.968 & $<0.001$ & 0.943 & $<0.001$ \\
\hline
\end{tabular}

a , compared with the same group before treatment $(\mathrm{P}<0.05) ;{ }^{\mathrm{b}}$, compared with the placebo group after treatment $(\mathrm{P}<0.05)$.

Table 6 Comparison of intervention efficacy of patients in the antibiotic group, microecological group, and placebo group [n (\%)]

\begin{tabular}{lcccc}
\hline Group & Significantly effective & Effective & \multicolumn{1}{c}{ Ineffective } & Total effective rate \\
\hline Antibiotic group $(\mathrm{n}=39)$ & $23(33.33)$ & $12(30.77)$ & $4(10.26)$ & 89.74 \\
Microecological group $(\mathrm{n}=36)$ & $19(52.78)$ & $14(38.89)$ & $3(8.33)$ & $16(61.54)$ \\
Placebo group $(\mathrm{n}=26)$ & $4(15.38)$ & $6(23.08)$ & & 31.67 \\
$\chi^{2}$-test & & & 29.959 & \\
P value & & & $<.001$ & \\
\hline
\end{tabular}

Table 7 Comparison of negative conversion rate of patients in the antibiotic group, microecological group, and placebo group [n (\%)]

\begin{tabular}{lcc}
\hline Group & $\begin{array}{c}\text { Negative } \\
\text { conversion }\end{array}$ & $\begin{array}{c}\text { Non-negative } \\
\text { conversion }\end{array}$ \\
\hline Antibiotic group $(\mathrm{n}=39)$ & $30(76.92)$ & $9(23.08)$ \\
Microecological group $(\mathrm{n}=36)$ & $31(86.11)$ & $5(13.89)$ \\
Placebo group $(\mathrm{n}=26)$ & $6(23.08)$ & $20(76.92)$ \\
$\chi^{2}$-test & \multicolumn{2}{c}{30.050} \\
P value & \multicolumn{2}{c}{$<0.001$} \\
\hline
\end{tabular}

\section{Comparison of symptom scores between the negative conversion and non-negative conversion groups after treatment}

The symptom score after treatment in the negative conversion group was $3.97 \pm 1.03$ points, and the symptom score after treatment in the non-negative conversion group was $5.85 \pm 1.24$ points. The difference between the 2 groups was statistically significant $(t=8.084, \mathrm{P}<0.001)$.

\section{Discussion}

IBS is currently the most common functional gastrointestinal disease. It is mainly characterized by abdominal pain, abdominal discomfort accompanied by bowel habits, and/or abnormal stool characteristics (10). The pathogenesis of the disease is complicated, the etiology is not clear, and there are no objective diagnostic criteria. Moreover, there are no effective means for treatment, and it is prone to recurrence, making it difficult for clinicians to treat (11).

SIBO is a group of clinical syndromes caused by abnormal types and quantities of bacteria in the small intestine due to various reasons. It mainly manifests as malabsorption of nutrients, abdominal distension, and diarrhea, and its symptoms are similar to those of IBS (12). It has been proposed in the literature that SIBO plays a major role in IBS, and IBS can be diagnosed indirectly by detecting SIBO, providing a new direction for the clinical treatment of IBS. At present, it is believed that the impact of SIBO on IBS mainly has the following aspects. First, abnormal intestinal motility. SIBO has a significant influence 
on the occurrence of abnormal intestinal motility. Bacterial overgrowth can slow down the transit of the small intestine and prolong the oral-blind transit time. Furthermore, studies have shown that IBS and SIBO can slow down phase III of the inter-digestive migratory compound movement, therefore, intestinal flora can produce different digestive symptoms by affecting intestinal motility (13). Second, high visceral sensitivity. Previous studies have suggested that hypersensitivity to mechanical, chemical, temperature, and electrical stimulation of the colon may be one of the most important pathogenic factors for IBS. In SIBO, the intestinal mucosal villi shows patchy widening and flattening, and intestinal permeability increases, resulting in a decrease in the colon's sensory threshold to irritation $(14,15)$. Third, SIBO activates the intestinal mucosal immune response. The normal intestine is colonized with a large number of bacteria, which interact with the body to maintain normal metabolism and various functions of the body, and at the same time stimulate the body to produce complete immune function. The overgrowth of bacteria will produce a variety of toxic substances. These substances, including bacteria themselves, can stimulate the intestinal immune cells to produce cytokines, which leads to increased sensitivity of the intestinal mucosa and induces intestinal irritability (16).

The results of the present study showed that the positive rate of SIBO in the IBS group was higher than that in the control group $(\mathrm{P}<0.05)$, suggesting that there is a correlation between SIBO and IBS. However, due to the similar symptoms of IBS and SIBO, detecting only the incidence of SIBO in IBS patients cannot indicate that SIBO is the cause of IBS, because SIBO may also be a symptom or complication of IBS (17). Therefore, we further observed improvements in the symptoms of IBS patients after the treatment of SIBO with antibiotics. The results showed that the patient symptoms of IBS and SIBO turned negative patient were significantly improved compared with those who did not turn from positive to negative. Therefore, the findings further indicate that that SIBO is the cause of IBS.

Inflammatory factors are soluble, low-molecularweight proteins produced by different cells induced by mitogens, immunogens, or other factors, and there are many reports on IBS and inflammatory factors in the literature (18). In the present study, we found that the level of pro-inflammatory factor IL- $1 \beta$ in the IBS group was significantly higher than that of the control group, but anti-inflammatory factor IL-10 was significantly lower than that of the control group $(\mathrm{P}<0.05)$, suggesting that inflammatory factors play a certain role in the pathogenesis of IBS. At the same time, IL- $1 \beta$ and IL-10 of SIBO patients were also compared. The results showed that the level of IL-1 $\beta$ in the SIBO-positive group was higher than that in the SIBO-negative group, while the IL-10 level was lower than that of the SIBO-negative group $(\mathrm{P}<0.05)$. After successful treatment of SIBO, the inflammatory factors in the negative group improved compared with those in the same groups before treatment, and the improvement was more significant than that in the non-negative group. This result further verified the conclusion that SIBO may be part of the pathogenesis of IBS (19). In summary, SIBO plays an important role in the occurrence of IBS. Clinically, antibiotics and microecological agents can be used to correct SIBO, thereby improving the symptoms of IBS patients and peripheral blood inflammatory factors.

\section{Acknowledgments}

Funding: None.

\section{Footnote}

Reporting Checklist: The authors have completed the STROBE reporting checklist. Available at http://dx.doi. org/10.21037/apm-21-427

Data Sharing Statement: Available at http://dx.doi. org/10.21037/apm-21-427

Conflicts of Interest: All authors have completed the ICMJE uniform disclosure form (available at http://dx.doi. org/10.21037/apm-21-427). The authors have no conflicts of interest to declare.

Ethical Statement: The authors are accountable for all aspects of the work in ensuring that questions related to the accuracy or integrity of any part of the work are appropriately investigated and resolved. All patients agreed to participate in this study and signed an informed consent form. This study was approved by the Sichuan Provincial People's Hospital, University of Electronic Science and Technology of China (No. 20180529). The study was conducted in accordance with the Declaration of Helsinki (as revised in 2013).

Open Access Statement: This is an Open Access article distributed in accordance with the Creative Commons 
Attribution-NonCommercial-NoDerivs 4.0 International License (CC BY-NC-ND 4.0), which permits the noncommercial replication and distribution of the article with the strict proviso that no changes or edits are made and the original work is properly cited (including links to both the formal publication through the relevant DOI and the license). See: https://creativecommons.org/licenses/by-nc-nd/4.0/.

\section{References}

1. Rao SSC, Bhagatwala J. Small Intestinal Bacterial Overgrowth: Clinical Features and Therapeutic Management. Clin Transl Gastroenterol 2019;10:e00078.

2. Ghoshal UC, Shukla R, Ghoshal U. Small Intestinal Bacterial Overgrowth and Irritable Bowel Syndrome: A Bridge between Functional Organic Dichotomy. Gut Liver 2017;11:196-208.

3. Rasmussen J, Duriancik DM. Management of Small Intestinal Bacterial Overgrowth in Adult Patients. Gastroenterol Nurs 2019;42:269-76.

4. Defrees DN, Bailey J. Irritable Bowel Syndrome: Epidemiology, Pathophysiology, Diagnosis, and Treatment. Prim Care 2017;44:655-71.

5. O'Mahony SM, Clarke G, Dinan TG, et al. Irritable Bowel Syndrome and Stress-Related Psychiatric Comorbidities: Focus on Early Life Stress. Handb Exp Pharmacol 2017;239:219-46.

6. Stefanolo J P TA, María M. Manresa. 858 - Therapeutic response to small intestinal bacterial overgrowth (SIBO) in irritable bowel syndrome (IBS). Is it useful to test for? Gastroenterology 2019;156:190-1.

7. Gwee KA, Ghoshal UC, Chen M. Irritable bowel syndrome in Asia: Pathogenesis, natural history, epidemiology, and management. J Gastroenterol Hepatol 2018;33:99-110.

8. Tziatzios G, Giamarellos-Bourboulis EJ, Papanikolaou IS, et al. Is small intestinal bacterial overgrowth involved in the pathogenesis of functional dyspepsia? Med Hypotheses 2017;106:26-32.

Cite this article as: $\mathrm{Yu} \mathrm{X,} \mathrm{Li} \mathrm{Y,} \mathrm{Xiang} \mathrm{F,} \mathrm{Feng} \mathrm{J.} \mathrm{Correlation}$ between small intestinal bacterial overgrowth and irritable bowel syndrome and the prognosis of treatment. Ann Palliat Med 2021;10(3):3364-3370. doi: 10.21037/apm-21-427
9. Ghoshal UC, Nehra A, Mathur A, et al. A meta-analysis on small intestinal bacterial overgrowth in patients with different subtypes of irritable bowel syndrome. J Gastroenterol Hepatol 2020;35:922-31.

10. Herndon CC, Wang YP, Lu CL. Targeting the gut microbiota for the treatment of irritable bowel syndrome. 2020;36:160-70.

11. Stanghellini V. Functional Dyspepsia and Irritable Bowel Syndrome: Beyond Rome IV. Dig Dis 2017;35 Suppl $1: 14-7$.

12. Pittman N, Rawn SM, Wang M, et al. Treatment of small intestinal bacterial overgrowth in systemic sclerosis: a systematic review. Rheumatology (Oxford) 2018;57:1802-11.

13. Mearin F, Rey E, Santander C. Irritable bowel syndrome: How to improve decision making in clinical practice. Med Clin (Barc) 2018;151:489-97.

14. Lacy BE. Patient-Centric Care of Diarrhea-Predominant Irritable Bowel Syndrome. J Fam Pract 2019;68:S43-8.

15. Hukkinen $M$, Kivisaari R, Koivusalo A, et al. Risk factors and outcomes of tapering surgery for small intestinal dilatation in pediatric short bowel syndrome. J Pediatr Surg 2017;52:1121-7.

16. Talley NJ, Holtmann G. Irritable bowel syndrome and functional dyspepsia: what can epidemiology tell us about etiology? Expert Rev Gastroenterol Hepatol 2018;12:633-5.

17. Beveridge C, Obelmejias R, Lynch KL. Su1761 small intestinal bacterial overgrowth: is it time to shorten testing? Gastroenterology 2020;158:S637.

18. Weinstock LB, Brook J, Kaleem Z, et al. 1194 Small Intestinal Bacterial Overgrowth Is Common in Mast Cell Activation Syndrome. Am J Gastroenterol 2019;114:S671.

19. Holster S, Brummer RJM, Repsilber D, et al. Fecal Microbiota Transplantation in Irritable Bowel Syndrome and a Randomized Placebo-Controlled Trial. Gastroenterology 2017;152:S101-2.

(English Language Editor: R. Scott) 